\title{
Giant paraesophageal hernia-related chronic gastric volvulus case to the emergent surgery
}

\author{
Hüseyin Çiyiltepe ${ }^{1}$, Ebubekir Gündeş ${ }^{1}$, Durmuş Ali Çetin ${ }^{1}$, Ulaş Aday ${ }^{1}$, Emre Bozdağ ${ }^{1}$, Fulya Çiyiltepe ${ }^{2}$ \\ ${ }^{1}$ Department of Gastroenterological Surgery, Kartal Kosuyolu High Speciality Education and Training Hospital, Istanbul, Turkey \\ 2Intensive Care Unit, Haydarpasa Education And Research Hospital, Istanbul, Turkey
}

Gastroenterology Rev 2017; 12 (4): 315-317

DOI: https://doi.org/10.5114/pg.2017.72111

Address for correspondence: Hüseyin Çiyiltepe, Department of Gastroenterological Surgery, Kartal Kosuyolu High Speciality Education and Training Hospital, 34000 Istanbul, Turkey, e-mail: drciyiltepe@hotmail.com

Gastric volvulus is a rare clinical condition defined as a rotation of all or a part of the stomach by at least $180^{\circ}$, which causes complete or partial obstruction. According to its aetiology it is classified as primary $30 \%$, type 1 ) or secondary (70\%, type 2). Type 1 gastric volvulus is often seen in childhood, and there is no underlying condition such as diafragmatic or intra-abdominal abnormality. Secondary gastric volvulus is usually seen in the elderly and is nearly always associated with paraesophageal and diaphragmatic hernias, diaphragm eventrations, or intra-abdominal adhesions [1, 2].

Anatomically it is divided according to the type of rotation. The rotation is defined as organoaxial if it is along the long axis of the stomach and mesenteroaxial if it is perpendicular to long axis of the stomach. Organoaxial type is encountered more frequently and is often associated with diaphragmatic hernia [3].

Some patients with secondary type gastric volvulus have no symptoms. According to the degree of rotation and rapidity of onset some patients present with intermittent vomiting, and if complete obstruction occurs patients present with Borchardt's triad of acute epigastric pain, severe retching without vomiting, and difficulty in nasogastric tube insertion. Acute gastric volvulus, ischaemia, and necrosis is a life-threatening condition that requires immediate diagnosis and treatment. Treating the underlying cause in chronic cases will prevent acute table [4].

In this case report we aim to present a patient with organoaxial gastric volvulus due to giant paraesophaegal hernia, who was admitted to hospital with cough and shortness of breath, and a follow-up period after laparoscopic treatment.

A 50-year-old woman was admitted to the chest diseases outpatient clinic with complaints of chronic cough and shortness of breath. The results of the chest chest X-ray revealed eventration of the left diaphragm and air presentation that was thought to belong to the stomach in the left hemithorax (Figure 1). There was no underlying diseases or history of trauma. The thoraco-abdominal computed tomography (CT) of the patient showed gastric volvulus presentation, formed secondarily to paraesopageal hernia that filled the left hemithorax almost completely, and volume loss in the right hemithorax related to the shifting of the mediastinum (Figure 2). The upper gastrointestinal contrast study also revealed that the esophagogastric junction was in its normal anatomical position and the whole stomach shifted in the thorax. The "upside-down stomach" (the rotation of the antrum towards the front and up and the rotation of the fundus towards the back and down), which was seen in the organo-axial gastric volvulus, was observed (Figure 3). Upper endoscopy results demonstrated that the lower oesophageal sphincter was at the level of the hiatal clamp but the antrum shifted upwards due to the rotational anomaly seen in the stomach, and the mucosa of the stomach and duodenum was normal. Endoscopic reduction was performed initially but emergency surgery was planned following the failure of the procedure and the emergence of acute symptoms. Intraoperational observation showed that the oesophageal junction had a normal location but that the whole stomach and a part of the transverse colonic wall were in the thorax due to paraesophageal hernia. The patient received laparoscopic mesh herniorrhaphy, Nissen fundoplication, and anterior suture gastropexy. The patient was discharged without any problems on post-op day 5 . The results of the patient's barium swallow test performed in postop month 3 (Figure 4) and the control endoscopy performed in the first year revealed that the stomach had normal anatomical localisation. 


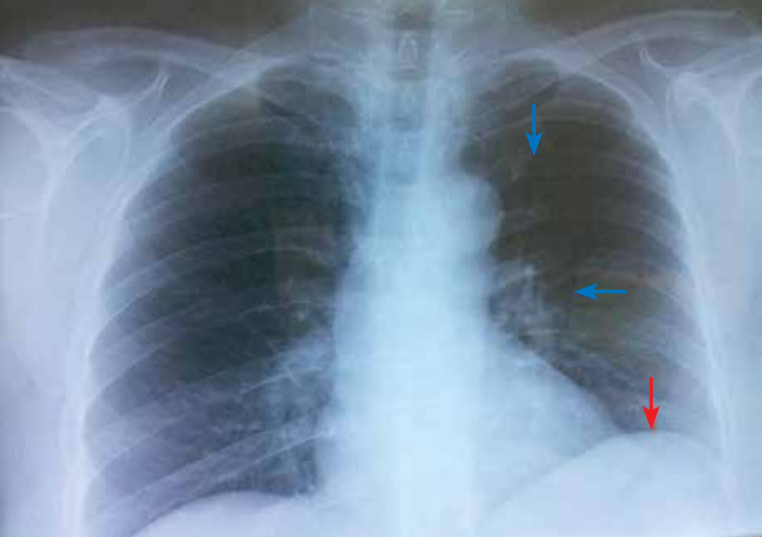

Figure 1. Chest X-ray showing the eventration of left diaphragm (red arrow) and air presentation in the left hemithorax (blue arrow)

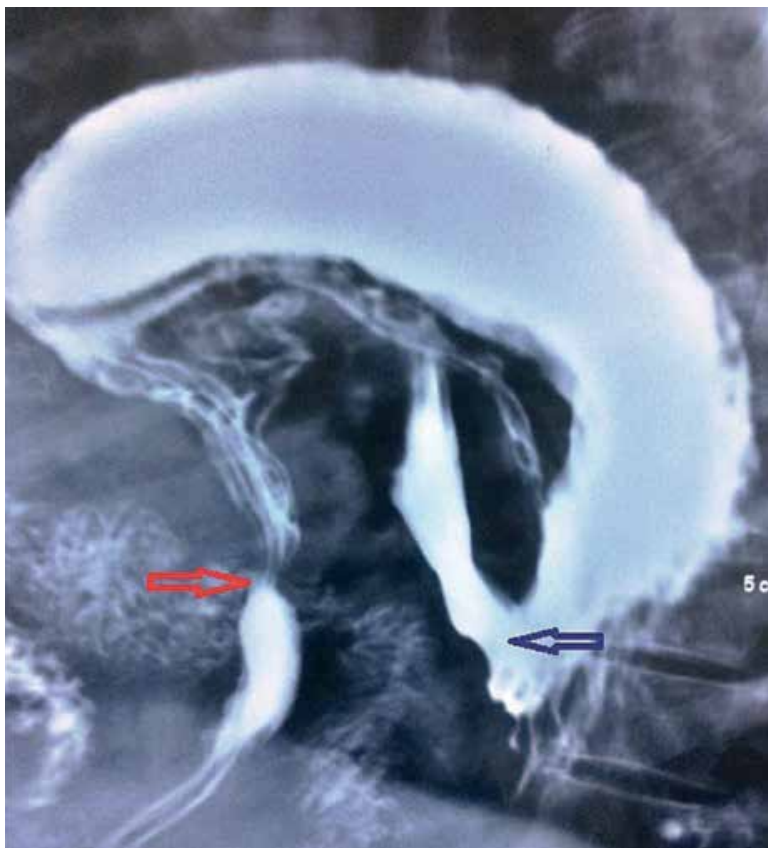

Figure 3. Upper gastrointestinal contrast study with "upside-down" stomach view (red arrow - pylorus, blue arrow - esophagogastric junction)

Although gastric volvulus is not frequently seen, it is a life-threatening clinical condition that might result in ischaemia/necrosis and related perforation in acute cases [5]. While it is aetiologically classified as primary or secondary, it is anatomically classified as organo-axial or meso-axial. There is no underlying diaphragmatic pathology in the first type, and it is more frequently seen in childhood. The secondary type generally develops secondarily to paraesophageal hernia or eventration of the diaphragm and is seen more frequently in adults [1, 2].

Studies in literature have reported that the mortality rates of volvulus were around $40-50 \%$. The clinical
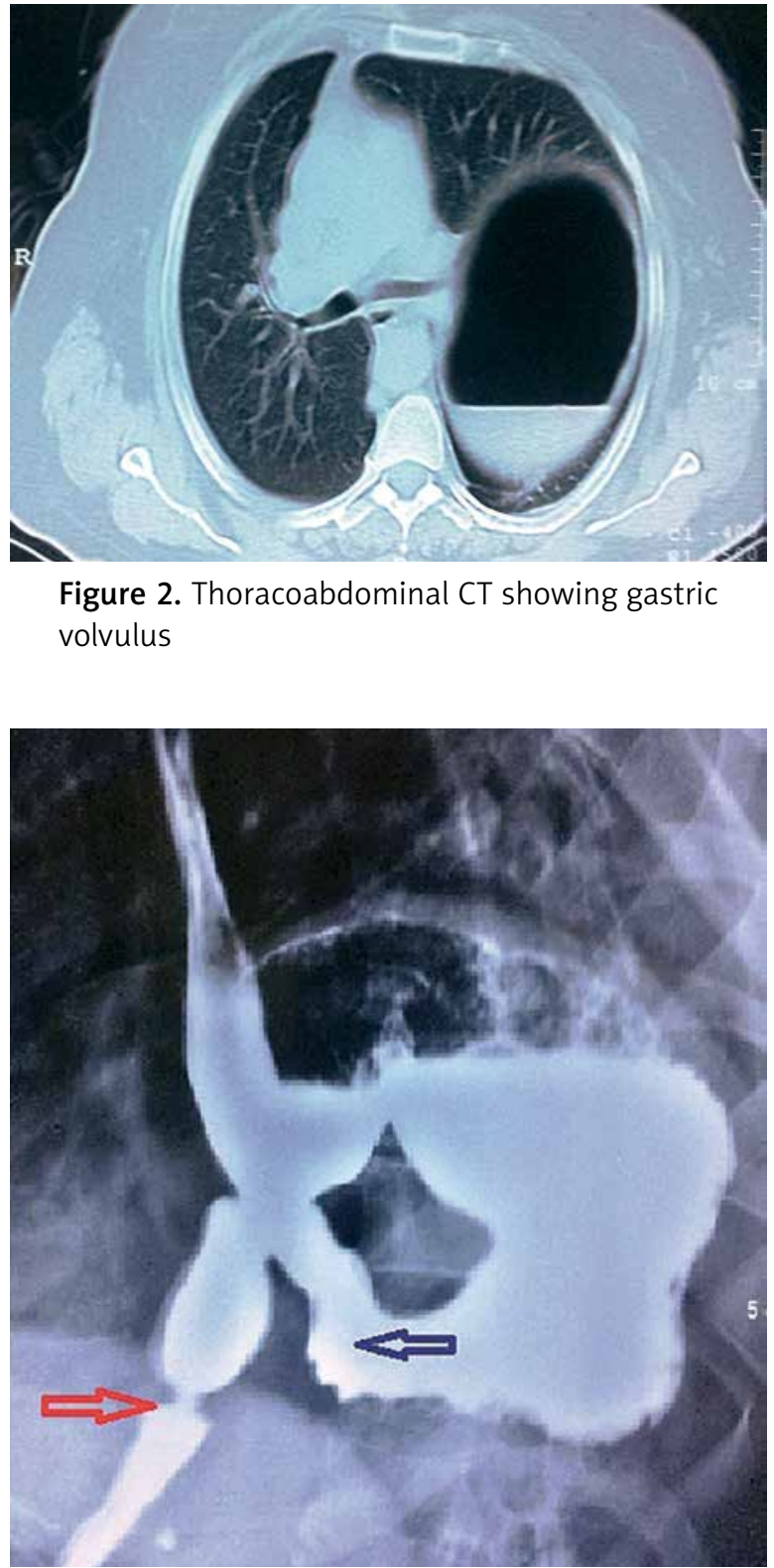

Figure 2. Thoracoabdominal CT showing gastric volvulus ture of acute cases is severe and patients should be taken into emergency surgery following diagnosis [6]. Chronic gastric volvulus, on the other hand, may have an asymptomatic progress. It may also present with existent hiatal hernia with respiratory distress related to the relocation of the fundus of the stomach or the stomach as a whole in the thorax other than symptoms like nonspecific dyspeptic complaints and vomiting related to the gastrointestinal system. As the symptoms of chronic gastric volvulus are more general and milder, what is significant is that the clinician suspects the condition and elicits diagnosis by way of imaging techniques [7]. 


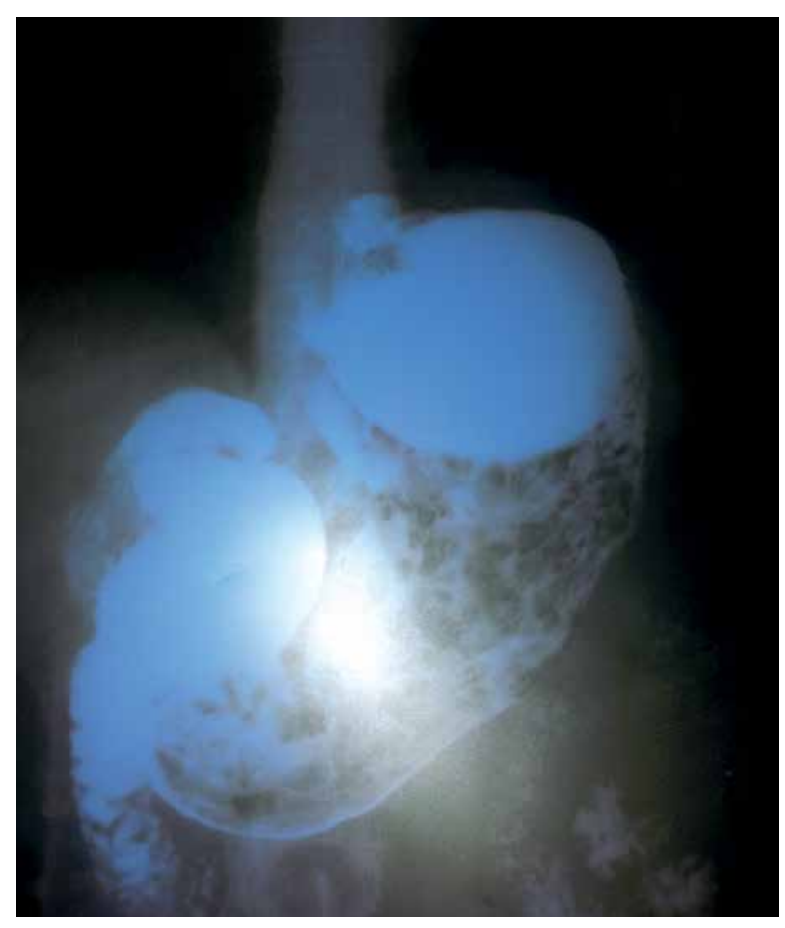

Figure 4. The upper gastrointestinal contrast study after three months of surgery

Currently accepted modes of treatment for gastric volvulus secondary to paraesophageal hernia are minimally invasive techniques. The goals of the surgical procedure in such cases are to relocate the herniated organs into the abdomen, to repair the present hiatal opening with mesh, and to perform fundoplication and/ or gastropexy in order to prevent recurrence [8]. In our case, the results of the barium swallow test performed in the third post-op month and the upper endoscopy performed in the first year revealed that the stomach was in its normal anatomical position.

Consequently, gastric volvulus is a rare clinical entity. It should be noted in the diagnosis of patients presenting to the emergency units with Borchardt's triad (epigastric pain, retching without vomiting, and failure to pass a nasogastric catheter). The chest X-ray should show gastric gas in the thorax and this should be confirmed by other techniques (thoracoabdominal CT, gastrointestinal contrast studies) within the framework of diagnosis. While emergency endoscopic reduction or surgical procedures are necessary for acute cases, surgery should also be performed in order to eliminate the symptoms and to prevent additional acute pathological conditions in chronic cases.

\section{Conflict of interest}

The authors declare no conflict of interest.

\section{References}

1. Godshall D, Mossallam U, Rosenbaum R. Gastric volvulus: case report and review of the literature. J Emerg Med 1999; 17: 837-40.

2. Teague WJ, Ackroyd R, Watson DI, Devitt PG. Changing patterns in the management of gastric volvulus over 14 years. $\mathrm{Br}$ J Surg 2000; 87: 358-61.

3. Naim HJ, Smith R, Gorecki PJ. Emergent laparoscopic reduction of acute gastric volvulus with anterior gastropexy. Surg Laparosc Endosc Percutan Techn 2003; 13: 389-91.

4. Palanivelu C, Rangarajan M, Shetty AR, Senthilkumar R. Laparoscopic suture gastropexy for gastric volvulus: a report of 14 cases. Surg Endosc 2007; 21: 863-6.

5. Lee HY, Park JH, Kim SG. Chronic gastric volvulus with laparoscopic gastropexy after endoscopic reduction: a case report. J Gastric Cancer 2015; 15: 147-50.

6. Smith RJ. Volvulus of the stomach. J Natl Med Assoc 1983; 75: 393-6.

7. Wu MH, Chang YC, Wu CH, et al. Acute gastric volvulus: a rare but real surgical emergency. Am J Emerg Med 2010; 28: 118-e5.

8. Jamil L, Huang B, Kunkel D, et al. Successful gastric volvulus reduction and gastropexy using a dual endo-scope technique. Case Rep Med 2014; 2014: 136381.

Received: 8.09.2016

Accepted: 6.10.2016 